\title{
RELATOS DE PESQUISAS
O CONHECIMENTO SOBRE A PLATAFORMA LATTES
(CNPq) NUMA PERSPECTIVA SISTÊMICA:
FUNDAMENTOS E LACUNAS PARA ESTUDOS EM
CIÊNCIA DA INFORMAÇÃO
}

Letícia Silvana dos Santos Estácio

Doutoranda em Ciência da Informação pela Universidade Federal de Santa

Catarina, Brasil.

E-mail: leticiasestacio@gmail.com

\section{William Barbosa Viana}

Doutor em Engenharia de Produção pela Universidade Federal de Santa Catarina, Brasil. Professor da Universidade Federal de Santa Catarina,

Brasil.

E-mail: wpwilliam@hotmail.com

\section{Vinicius Medina Kern}

Doutor em Engenharia de Produção pela Universidade Federal de Santa Catarina, Brasil. Professor da Universidade Federal de Santa Catarina, Brasil.

E-mail: vmkern@gmail.com

\begin{abstract}
Resumo
O objetivo do artigo é identificar um portfólio bibliográfico básico, relevante e interdisciplinar sobre a Plataforma Lattes com vistas ao desenvolvimento de estudos que a considerem como fonte de informação numa perspectiva sistêmica. As Plataformas de Dados Web of Science e Scopus foram eleitas para a busca de artigos científicos relacionados ao tema de pesquisa por sua amplitude e representatividade. Na primeira etapa foram definidas as palavras-chave "Lattes Platform", "Systemism", "Plataform-government" e "Information Systems" e a seleção do portfólio foi baseada numa visão sócio-técnica. Os resultados identificaram um conjunto de artigos interdisciplinares, periódicos e autores que fundamentam os estudos sobre o assunto, bem como evidenciaram uma importante lacuna para Ciência da Informação que é a carência de estudos que considerem a plataforma como objeto de estudo na visão de seus usuários.
\end{abstract}

Palavras-chave: Gestão da Informação. Plataforma Lattes. Abordagem sistêmica.

\section{THE KNOWLEDGE ON THE LATTES PLATFORM (CNPq) IN A SYSTEMIC PERSPECTIVE: FUNDAMENTALS AND GAPS FOR STUDIES IN INFORMATION SCIENCE}

\begin{abstract}
The objective of this article is to identify a basic, relevant and interdisciplinary bibliographic portfolio on the Lattes Platform with a view to developing studies that consider it as a source of information from a systemic perspective. The Web of Science and Scopus Data Platforms were chosen for the search of scientific papers related to the topic of research for their breadth and representativeness. In the first stage the keywords "Lattes Platform", "Systemism", "Plataform-government" and "Information Systems" were defined and the portfolio selection was based on a socio-technical view. The results identified a set of interdisciplinary articles, periodicals and authors that base the studies on the subject, as well as evidenced an important gap for Information Science that is the lack of studies that consider the platform as object of study in the view of its users.
\end{abstract}

Keywords: Information Management. Platform Lattes. Systemic approach.

Perspectivas em Gestão \& Conhecimento, João Pessoa, v. 9, n. 1, p. 198-211, jan./abr. 2019. DOI: http://dx.doi.org/10.21714/2236-417X2019v9n1p198

http://periodicos.ufpb.br/ojs2/index.php/pgc. ISSN: 2236-417X. Publicação sob Licença (cc) EY-NC-ND 


\section{INTRODUÇÃO}

O uso de intensivo de informação em todas as esferas da vida social tem impactado na forma como as organizações interagem com os cidadãos, incluídos os governos, que necessitam se modernizar e incrementar o uso de novas tecnologias de informação e comunicação (TICS) para o acesso a dados e informações públicas em seus Sistemas de Informação (SI).

Considera-se Sistema de Informação um conjunto organizado de pessoas, hardware, software, redes de comunicações e recursos de dados que coleta, transforma e dissemina informações em uma organização (O'Brien, 2004, p. 49).

O surgimento da web na década de 80 e a integração de novas ferramentas e métodos da tecnologia da informação permitiram melhorias e impactos significativos nas formas de comunicação, principalmente, nos sistemas de informação (SI) em ciência e tecnologia governamentais, incluindo-se as denominadas plataformas e-gov, portais de transparência, dados abertos e assim por diante. Esses SI utilizam recursos tecnológicos computadorizados afim de coletar, recuperar, processar, armazenar e distribuir dados e informações, ou seja, funcionam como mecanismos de suporte, acesso e fonte de informação.

Recentes estudos das Organizações das Nações Unidas (ONU) em 2016 deram conta de informar que países com alto nível de desenvolvimento socioeconômico apresentam os melhores resultados em termos de e-gov, considerando serviços on-line, capital humano e infraestrutura de telecomunicação, sendo que o Brasil ocupou a 51 a posição, muito atrás de outros países latino-americanos, como Uruguai $\left(34^{\circ}\right)$, Argentina $\left(41^{\circ}\right)$ e Chile $\left(42^{\circ}\right)$.

No Brasil, voltada à gestão de Ciência Tecnologia \& Inovação (CT\&I) a Plataforma Lattes é uma plataforma do governo (e-gov) que constitui um conjunto de $\mathrm{SI}$, base de dados e portais web que promovem a integração dos SI das agências federais, a fim de racionalizar o processo de gestão da Ciência \& Tecnologia (C\&T) tanto do ponto de vista do usuário quanto das agências de fomento e das instituições de ensino e pesquisa do país.

A Plataforma Lattes representa a "integração de seus sistemas de informação gerenciais, instrumento fundamental não somente para as atividades de financiamento, mas também no tratamento e na difusão de informações necessárias para a formulação e a gestão de políticas de ciência e tecnologia" (SABBATINI, 2001).

A ideia que se tem sobre a Plataforma Lattes, mais especificamente sobre o Sistema de Currículo Lattes é de que sua função torna-se de grande importância para a comunidade científica diante da divulgação de suas produções, áreas do conhecimento, interesse, projetos de pesquisas dentre outros. Por outro lado, fatores como investimento podem influenciar na manutenção e atualização da plataforma em razão da mesma pertencer a órgão público, o qual fatores políticos e econômicos podem pesar nas tomadas de decisões.

A Plataforma Lattes interage com um conjunto de sub-sistemas que proporcionam suporte à captação e manutenção de dados, seja do sistema de Currículos, Grupos de Pesquisa e Instituições de Ensino e Pesquisa, sendo considerada uma relevante fonte de informação no contexto de C\&T no país, beneficiando seus usuários na tomada de diversos tipos de decisão.

No caso, esse SI é constituído não apenas por recursos tecnológicos como também por atores, usuários, instituições, agências de fomento e entre outros fatores sociais, que o influenciam ou recebem sua ação, podendo agir de maneira positiva ou negativa no funcionamento da Plataforma e seus sub-sistemas, como por exemplo, o Sistema de Currículo Lattes.

Os estudos de sistemas complexos, como se pode considerar o da Plataforma Lattes, requerem abordagens amplas e sistêmicas de forma a compreender seu funcionamento tanto técnico quanto social e identificar pontos críticos para sua melhoria. Nesse contexto, o

Perspectivas em Gestão \& Conhecimento, João Pessoa, v. 9, n. 1, p. 198-211, jan./abr. 2019 
funcionamento dos SI depende de um sistema técnico que interage com um sistema social, ou seja, há que considerar a colaboração de agentes humanos e artificiais é essencial para a emergência de propriedades informacionais dos SI.

Tal é sinalizado por Castells (2005, p. 349), para quem primeira fase da sociedade da informação se caracterizou pelo desenvolvimento da tecnologia e a segunda deu enfoque à assuntos sociais, como "ênfase à mudança da forma como trabalhamos.

$O$ autor ainda informa que o uso das TICs pelo Estado passa a ser visto como um papel decisivo no desenvolvimento e moldagem da sociedade em rede uma vez que não são as tecnologias que determinam a sociedade, mas a sociedade é que dá a forma às tecnologias segundo sua necessidade, induzindo governos a se adaptarem ao uso das já introduzidas no cotidiano das pessoas (Castells, 2005).

Nesse sentido, considerando a importância tanto de fatores técnicos como sociais para o desenvolvimento de concepções e melhorias na Plataforma Lattes para promover automatização de processos, padronização de procedimentos e ampliação no fluxo de informações, esse estudo busca identificar na literatura o que se tem publicado sobre o assunto, na busca de um referencial que norteie futuros estudos numa perspectiva sistêmica.

Para tanto, pergunta-se: o que tem sido publicado no Brasil e no Exterior sobre a Plataforma Lattes nesta década?

O objetivo é identificar um Portfólio Bibliográfico básico, relevante e interdisciplinar sobre o assunto que possibilite a uma fundamentação consistente para futuros estudos a partir do conhecimento já construído com vistas à consideração da Plataforma Lattes como fonte de informação numa perspectiva sistêmica e sócio-tecnica.

As Plataformas de Dados Web of Science e Scopus foram eleitas para a busca de artigos científicos relacionados ao tema de pesquisa por sua amplitude e representatividade. $\mathrm{Na}$ primeira etapa foram definidas as palavras-chave "Lattes Platform", "Systemism", "Plataformgovernment" e "Information Systems". Ao realizar a primeira busca em ambas as bases, foram adicionadas em cada campo de pesquisa as palavras-chave conforme descritas acima, utilizando como estratégia de busca o operador booleano "AND" a fim de recuperar documentos com os termos estabelecidos, além disso, o uso de aspas (") foram aplicadas com o objetivo de recuperar os termos exatamente iguais os de busca.

Nas buscas foi delimitada uma cobertura temporal dos artigos entre os anos de $2010 \mathrm{e}$ 2016, bem como as áreas de conhecimento Social Sciences \& Humanities (Scopus) e Information Science/Library Science (Web of Science), além disso, a preferência do tipo de documento concentrou-se apenas em artigos, eliminando-se outros tipos de documentos.

$\mathrm{Na}$ base de dados da Web of Science, optou-se por selecionar o item "Tópico" para realizar a busca, ou seja, sendo possível recuperar documentos em que, a palavra-chave inserida esteja em qualquer parte do conteúdo no texto. Na Scopus foi selecionado o item "Article Title, Abstract, Keywords", na escolha desta opção as palavras-chave deverão estar contidas no título do artigo, resumo e palavras-chave.

Considerando-se na análise qualitativa que selecionou o portfólio, o 'lugar do sujeito epistêmico', ou seja, a maneira de abordar o sujeito da pesquisa proposta dar-se-á por meio do construtivismo, no qual o conhecimento é o resultado de algum tipo de interação entre um sujeito e um objeto. Nesse sentido, o objeto da pesquisa consiste na Plataforma Lattes, mais precisamente, o Sistema de Currículo Lattes, permitindo a intervenção do sujeito no sentido de direcionar o olhar ou a ação ao objeto (título, abstract e conteúdo dos artigos) a partir de identificar os que tratavam dos mecanismos que promovem e obstruem o funcionamento da plataforma.

O principal fundamento teórico para uma visão integrada do sistema tecnológico e social foi a perspectiva do sistemismo de Mario Bunge, considerado uma visão de mundo que

Perspectivas em Gestão \& Conhecimento, João Pessoa, v. 9, n. 1, p. 198-211, jan./abr. 2019 
pode ser utilizada como base nos estudos empíricos de qualquer sistema, incluindo sistemas técnicos e sociais.

\section{ASPECTOS TEÓRICOS NORTEADORES DA SELEÇÃO BIBLIOGRÁFICA}

Considerando o tema propostos neste estudo, observa-se a utilidade de uma relação interdisciplinar do campo da Ciência da Informação $(\mathrm{Cl})$ com outras áreas do conhecimento. Sob o ponto de vista teórico e epistemológico, Japiassu (1976, p.44) aponta que as pesquisas interdisciplinares podem surgir de preocupações "relativas às estruturas e aos mecanismos comuns às diferentes disciplinas científicas que são chamadas a ingressar num processo de interação ou colaboração".

Para Pinheiro (1998, p.14) as relações interdisciplinares "são relações de troca teórica e metodológica", para que tal ocorra, tornando-se relevante a percepção no momento em que as disciplinas envolvidas se encontram.

No campo da $\mathrm{Cl}$, a interdisciplinaridade é discutida desde sua origem em vista das interações que estabelece com outras áreas de conhecimento, e também, com a incorporação de conceitos, técnicas e teorias. Hawkins et al. (2003) a definem como um campo interdisciplinar com conceitos teóricos e práticos, bem como tecnológicos, legais e industriais, que lida com a transferência de conhecimentos, procurando satisfazer as necessidades informacionais.

Para o tema proposto por esse artigo e suas relações interdisciplinares, pode-se ainda consultar os autores dispostos no Quadro 1:

Quadro 1 - Autores, áreas e conceitos para embasamento dos aspectos teóricos

\begin{tabular}{|c|c|c|}
\hline Autores & Áreas & Conceitos \\
\hline Harold Borko & \multirow{5}{*}{ Ciência da Informação } & Informação \\
\hline Chaim Zins & & Interdisciplinaridade \\
\hline Carlos Alberto Ávila Araújo & & Ciência da Informação \\
\hline Patrícia Maria Silva & & Sistemas de Informação \\
\hline Lena Vânia Ribeiro Pinheiro & & Teoria Sistêmica \\
\hline Vinícius Medina Kern & \multirow{2}{*}{ Engenharias } & E-government (plataformas) \\
\hline Roberto Pacheco & & Sistemas de Informação \\
\hline Florência Ferrer e Paula Santos & \multirow{3}{*}{ Administração } & E-government \\
\hline Pedro Luiz Côrtes & & \multirow{2}{*}{ Sistemas de Informação } \\
\hline Jayr Figueiredo de Oliveira & & \\
\hline Mario Bunge & Filosofia & Sistemismo \\
\hline
\end{tabular}

Fonte: Os autores (2017)

Os autores apresentam estudos que servem de suporte teórico para abordagens interdisciplinares a partir de quatro área de conhecimento e palavras-chave relacionadas aos assuntos: $\mathrm{Cl}$, intersiciplinaridade, sistemas de informação e E-government.

Definida por Borko (1968, p. 3) como a "disciplina que investiga as propriedades e o comportamento da informação, as forças que regem o fluxo informacional e os meios de processamento da informação para a optimização do acesso e uso" a $\mathrm{Cl}$ relaciona-se com:

Perspectivas em Gestão \& Conhecimento, João Pessoa, v. 9, n. 1, p. 198-211, jan./abr. 2019 
a origem, coleta, organização, armazenamento, recuperação, interpretação, transmissão, transformação e utilização da informação. Isto inclui a investigação, as representações da informação tanto no sistema natural, como no artificial, o uso de códigos para uma eficiente transmissão de mensagens e o estudo dos serviços e técnicas de processamento da informação e seus sistemas de programação.

A partir desta ampla definição da área, diversos outros autores realizaram estudos a fim de discutir e reelaborar um novo conceito. Zins (2007) focou em explorar os significados da $\mathrm{Cl}$ e como ela é entendida na visão de pesquisadores da área, sendo possível criar uma relação de conceitos para descrevê-la, dentre eles, relacionado à temática desta pesquisa, a $\mathrm{Cl}$ é destacada como a:

ciência dos sistemas de informação, a qual estuda a informação (como um processo, como produto ou como um estado de consciência), bem como os seus cinco sub-processos básicos - geração, processamento, comunicação, armazenamento e uso, de modo que possa proporcionar acesso à informação de forma eficaz e/ou eficiente. Seu objetivo é facilitar a transmissão do conhecimento de uma pessoa para outra e a partir de uma geração para a outra, a fim de acelerar o progresso da humanidade (ZINS, 2007, p. 337).

Com base no estudo das correntes teóricas da Cl, Araújo (2009) aponta que, ao longo do desenvolvimento do campo, diversas foram as áreas e subáreas específicas que surgiram e se consolidaram. Dentre elas, a Teoria Sistêmica, correlata a um dos temas a ser estudado nesta pesquisa. Para Araújo (2009, p. 195) essa "teoria ganha expressão no campo da $\mathrm{Cl}$ com a publicação do trabalho de Wiener (1948) sobre a cibernética".

A origem de estudos sobre os sistemas acontece por volta de 1950, impulsionado pelo biólogo alemão Bertalanffy o qual atribui o nome de Teoria Geral dos Sistemas. Suas pesquisas estão voltadas ao comportamento dos organismos vivos, constatando-se que, diante da variedade de formas e características, os organismos possuíam pontos em comum (Oliveira, 2004; Rezende; Abreu, 2003; Stair; Reynolds, 2002).

E numa perspectiva sócio-técnica, o sistema é aberto e interage com o ambiente, é capaz de auto-regulação e pode alcançar um mesmo objetivo a partir de diferentes caminhos e usando diferentes recursos. É formada por dois subsistemas: o subsistema técnico - que são as máquinas, equipamentos, técnicos e o subsistema social - que são os indivíduos e grupos de indivíduos, seus comportamentos, capacidades, cultura e sensibilidade (BERTALANFFY, L. V., 1969).

$\mathrm{Na}$ área da $\mathrm{Cl}$, a teoria sistêmica da informação possui uma significativa expressão no campo com possibilidade de ampliação e sua contribuição à área apresenta-se por meio de duas grandes manifestações; a primeira - relacionada à função da informação na sociedade de forma que as unidades informacionais promovem o acesso à mesma por meio de repositórios de dados os quais servem de complemento para adquirir novos conhecimentos. A segunda manifestação - relaciona-se com o desenvolvimento das teorias sobre SI, buscando caracterizar os processos adequados para o funcionamento dos SI sob a lógica de processos.

Silva (2008, p. 258) considera que os SI no campo da Cl baseiam-se em

informações, ou seja, a sua matéria prima é a informação, podendo ser tecnicamente definido como um conjunto de componentes interrelacionados que coleciona ou recupera, processa e distribui informação. 0 sistema de informação pode ser manual ou computadorizado, e, nos dois casos, o elemento indispensável ao bom funcionamento do sistema é o

Perspectivas em Gestão \& Conhecimento, João Pessoa, v. 9, n. 1, p. 198-211, jan./abr. 2019 
indivíduo, pois é ele que concebe, programa, gerencia, executa e mantém o sistema; assim, a partir desse processo, existirá a seleção e a organização das informações para a sua efetiva utilização.

Na visão de Côrtes (2008, p. 1) o elemento principal de um SI é a informação, os objetivos destes sistemas consistem em armazenar, tratar e fornecer informações, a fim de que auxilie no apoio de determinadas funções e processos de instituições, agências, centros, universidades e organizações de modo geral. Para o autor, um SI pode ser "composto de um sub-sistema social (inclui as pessoas, processos, informações e documentos) e de um subsistema automatizado (máquinas, computadores, redes de comunicação) que interligam os elementos do sub-sistema social".

Nesse sentido, a popularização do computador e, mais tarde, da Internet contribuíram para o avanço e uso dos SI como uma das tecnologias de informação e comunicação utilizadas pelo homem, permitindo a imposição pela sociedade para com os governos sobre a modernização, transparência e efetividade de sua gestão, sendo que, a disponibilização de informações torna-se um recurso indispensável para o funcionamento das relações sociais.

Diante o exposto, o comportamento dos órgãos púbicos e privados vem seguindo tendências das novas tecnologias da informação e comunicação incorporando-as no seu dia-adia e, consequentemente, provocando mudanças significativas em seus ambientes de trabalho.

Por sua vez, o Governo de diversos países tem procurado se valer desses novos recursos por meio de portais eletrônicos, denominados (e-government/e-gov/governo eletrônico), a fim de "atender as expectativas da sociedade, a partir do acesso tecnológico, com o objetivo de facilitar e integrar a participação da comunidade na gestão pública" (MATSUDA, 2006, p. 10).

Segundo Koh et al. (2005), e-gov pode ser definido como o uso da Internet e de tecnologias da informação para simplificar ou melhorar o método pelo qual cidadãos, funcionários, parceiros e outras organizações de governo interagem e realizam negócios.

Para o Pacific Council on International Policy, (2002, p.56, tradução nossa) o e-gov compreende o "[...] uso da tecnologia da informação e da comunicação para promover maior eficiência e maior efetividade governamental, facilitando o acesso aos serviços públicos, permitindo ao grande público o acesso à informação, e tornando o governo mais responsável para com o cidadão".

Conforme Jardim (2000, p. 4), [...] o governo eletrônico expressa uma estratégia pela qual o aparelho de Estado faz uso das novas tecnologias para oferecer à sociedade melhores condições de acesso à informação e serviços governamentais, ampliando a qualidade desses serviços e garantindo maiores oportunidades de participação social no processo democrático.

Contudo, projetos e-gov podem ser vetores do avanço em direção à sociedade do conhecimento, entretanto, torna-se necessário que gestores públicos tenham consciência e valorizem o potencial transformador de uma plataforma e-gov.

Para Pacheco, Kern e Steil (2007, p. 72) a fim de "viabilizar o compartilhamento de informações e o estabelecimento de espaços de cooperação, as soluções e-gov devem ser abertas, flexíveis, robustas e, sobretudo, abrangendo o interesse de todos os atores afetos ao domínio para o qual a plataforma e-gov está sendo desenvolvida".

Ferrer e Santos (2004) relatam que, no Brasil, em 1996 a expressão Governo Eletrônico passou a ser utilizada para divulgar os serviços eletrônicos disponíveis pelo governo federal. Em dezembro de 1999, durante o mandato do presidente Fernando Henrique Cardoso foi aprovada a oferta pela internet de todos os serviços prestados ao cidadão, dando assim, lançamento ao Programa Sociedade da Informação que reuniu as estratégias do Governo

Perspectivas em Gestão \& Conhecimento, João Pessoa, v. 9, n. 1, p. 198-211, jan./abr. 2019 
Eletrônico e os aspectos sociais de inclusão digital para as atividades relacionadas à tecnologia de informação do país.

No Brasil, desenvolvida pelo CNPq órgão subordinado ao Ministério da Ciência, Tecnologia, Inovações e Comunicação, a Plataforma Lattes é um dos exemplos de projetos $e$ gov, atendendo a um domínio de interesse público, relacionado à CT\&I, reunindo informações e gerando novos conhecimentos sobre o sistema científico-tecnológico do país.

Atualmente, a porção mais aparente da Plataforma Lattes é o Sistema de Currículo Lattes, considerado um banco de informações curriculares mantidos pelo CNPq, tonando-se uma referência nacional para os profissionais de nível superior no Brasil. Para estes profissionais que possuem currículo e movimentam a Plataforma Lattes é um diferencial para quem pretende ingressar no mercado de trabalho empresarial, para quem pretende seguir a carreira acadêmica, servir de pré-requisito para se candidatarem às bolsas de pesquisas, financiamento de projetos, processos seletivos. Contemplando o registro de suas produções científica e tecnológica em âmbito nacional e internacional, além de identificar suas áreas temáticas de interesse, linhas de pesquisa e projetos.

O Diretório do Grupo de Pesquisas do CNPq compõe um dos SI da Plataforma Lattes, considerado um significativo instrumento na troca de informações pela comunidade científica e tecnológica a respeito do que se investiga quem investiga e onde se investiga.

Segundo Brito, Quonian e Mena-Chalco (2016) a Plataforma Lattes é uma ferramenta estratégica para planejamento e gestão, resultado da iniciativa do Conselho Nacional de Desenvolvimento Científico e Tecnológico (CNPq), para integrar em um ambiente disponível online as principais agências de fomento federais e estaduais a uma base de dados composta por Currículos, Grupos de Pesquisa e Instituições em um único Sistema de Informações.

E o Currículo Lattes, um dos recursos desta plataforma com histórico das atividades e produções de pesquisadores no Brasil, utilizadas como critérios avaliativos para instituições como a CAPES (Coordenação de Aperfeiçoamento de Pessoal de Nível Superior) e outros órgãos de pesquisa; seleção de estudantes e candidatos a bolsas e auxílios; avaliação de cursos de Pós-Graduação; seleção de corpo docente em processos seletivos; acompanhamento da produção científica e tecnológica nacional, dentre outras funções (BRITO, A.; QUONIAN, L.; MENA-CHALCO J. , 2016).

Desse modo, percebe-se a relevância da inserção da criação de novos grupos, bem como a atualização de informações, considerando a necessidade de se ter o conhecimento do que se faz em termos de investigação no país. Assim como Diretório do Grupo de Pesquisas, a Plataforma Lattes conta também com mais um SI, sendo reconhecido como o Diretório de Instituições, dispondo de dados cadastrais das instituições do Sistema Nacional de CT\&I do país, oportunizando que estas atualizem suas próprias informações.

Para Bunge (2003, p.114) tudo é sistema, "toda coisa, seja concreta ou abstrata, é um sistema ou um componente ou potencial componente de sistema". Partindo desse princípio, o estudo de sistemas, deve buscar a compreensão do todo e de suas relações com os indivíduos ou partes que o compõem.

Nesse sentido, a visão de que o conceito de sistema é central para a ciência e a tecnologia social e, com efeito, para todas as ciências e tecnologias, pode ser chamada sistemismo. O sistemismo tem dois componentes: ontológico e metodológico. O sistemismo ontológico afirma que o mundo é um sistema antes que uma coleção de indivíduos ou um bloco sólido. Seu parceiro metodológico é a visão de que os todos (wholes) são mais bem entendidos por meio da análise (top-down) e indivíduos por meio da síntese (bottom-up) (BUNGE, 2006, p. 12-13, tradução nossa).

O sistemismo fundamenta-se em cinco postulados: a) toda coisa, seja concreta ou abstrata, é um sistema ou um componente ou potencial componente de sistema; b) sistemas têm características sistêmicas (emergentes) que seus componentes não têm; c) todos os 
problemas deveriam ser abordados de forma sistêmica em vez de em forma fragmentada; d) todas as ideias deveriam ser unidas em sistemas (preferencialmente teorias); e) o teste de qualquer coisa, seja ideia, método ou artefato, supõe a validade de outros itens que são tomados como pontos de referência (benchmark) provisoriamente. f) Esses postulados sustentam a visão sistêmica de Bunge. Fornecem os fundamentos ontológicos e também apontam diretrizes metodológicas, mas não são suficientes para modelar sistemas (BUNGE, 2000).

Um sistema concreto pode ser descrito segundo o modelo CESM (Composition, Environment, Structure, Mechanism): a) Composição é a coleção de partes ou elementos componentes; b) Entorno ou Ambiente é a coleção de itens externos que atuam ou sofrem ação por algum componente; c) Estrutura é a coleção de ligações entre componentes e entre esses, e, itens do ambiente.

As ligações entre componentes formam a endoestrutura e as ligações entre componentes e itens do ambiente formam a exoestrutura; d) Mecanismo é a coleção de processos que geram a novidade qualitativa, isto é, que promovem ou obstruem as transformações, causando a emergência, crescimento, manutenção ou o desmantelamento do sistema ou de alguma de suas propriedades (BUNGE, 1997).

Considerado uma visão de mundo, o sistemismo pode ser utilizado como base nos estudos empíricos de qualquer sistema, incluindo sistemas técnicos e sociais, sendo esse o caso da Plataforma Lattes cujo funcionamento depende de um sistema técnico que interage com um sistema social.

\section{ASPECTOS METODOLÓGICOS E RESULTADOS}

A fim de descobrir resposta para alguma indagação, a pesquisa se define como uma atividade que se utiliza de métodos e pressupostos para alcançar seus objetivos (VOLPATO, 2004). Para conduzi-la, é necessário pensar em sua estrutura, que apresentará "o caminho do pensamento e a prática exercida na abordagem da realidade" (MINAYO, 2010, p. 14).

Neste primeiro momento da pesquisa, busca-se por meio de uma revisão sistemática utilizar "como fonte de dados à literatura sobre determinado tema. Esse tipo de investigação disponibiliza um resumo das evidências relacionadas a uma estratégia de intervenção específica, mediante a aplicação de métodos explícitos e sistematizados de busca, apreciação crítica e síntese da informação selecionada" (SAMPAIO; MANCINI, 2007, p. 84).

O resultado dos dados relacionados às palavras-chave "Lattes Platform", "Systemism" e, "Plataform-government" corresponderam à expectativa de uma busca precisa. Entretanto, os resultados referentes à palavra-chave "Information Systems", excedeu o limite de documentos para a realização de uma possível análise do que se trata relevante ou não. Sendo assim, optou-se por agrupar esta palavra com outras (Information Systems and Lattes Platform, Plataform-government and Lattes Platform e Information Systems and Plataformgovernment), restringindo assim a busca por conteúdos de interesse.

De acordo com as etapas de busca descritas acima, o Quadro 2 apresenta a distribuição de documentos recuperados por palavras-chave e base de dados.

Quadro 2 - Documentos recuperados por palavras-chave e base de dados

\begin{tabular}{|c|c|c|}
\hline Palavras-chave & Web of Science & Scopus \\
\hline Lattes Platform & 10 & 14 \\
\hline Systemism & 7 & 4 \\
\hline
\end{tabular}

Perspectivas em Gestão \& Conhecimento, João Pessoa, v. 9, n. 1, p. 198-211, jan./abr. 2019 


\begin{tabular}{|c|c|c|} 
Plataform-government & 25 & 50 \\
\hline Information Systemsand Lattes Platform & 3 & 4 \\
\hline Plataform-government and Lattes Platform & 0 & 0 \\
\hline Information Systems and Plataform-government & 9 & 0 \\
\hline Totais & $\mathbf{5 4}$ & $\mathbf{7 2}$ \\
\hline
\end{tabular}

Fonte: Os autores (2017)

A última etapa consistiu em organizar as bibliografias recuperadas por meio do software EndNote ${ }^{\circledR}$, nesse, foram eliminadas as bibliografias duplicadas, bem como as que não estavam disponíveis para acesso. Sendo assim, das 126 bibliografias recuperadas em ambas as bases, 43 bibliografias eram duplicadas e não disponíveis para acesso, 33 bibliografias foram selecionadas por meio da leitura do título, resumo e palavras-chave de cada artigo, esta escolha deu-se por meio da leitura do título, resumo e palavras-chave de cada artigo, por último, 58 delas foram desconsideradas por levar em consideração que as mesmas não eram relevantes para pesquisa. Conforme o Quadro 3, segue relação das 25 bibliografias selecionadas para a realização da leitura do conteúdo na íntegra.

Quadro 3 - Bibliografias selecionadas nas bases Web of Science e Scopus (2010-2016)

\begin{tabular}{|c|c|c|c|}
\hline Autor & Título & Periódico & Ano \\
\hline Bunge, $\mathrm{M}$. & $\begin{array}{l}\text { Social mechanisms and mechanismic } \\
\text { explanation }\end{array}$ & $\begin{array}{l}\text { BERLINER JOURNAL FUR } \\
\text { SOZIOLOGIE }\end{array}$ & 2010 \\
\hline Thalos, M. & Two Conceptions of Fundamentality & $\begin{array}{l}\text { PHILOSOPHY OF THE SOCIAL } \\
\text { SCIENCES }\end{array}$ & 2011 \\
\hline $\begin{array}{l}\text { Ranerup, A. et. } \\
\text { al. }\end{array}$ & $\begin{array}{c}\text { The socio-material pragmatics of e- } \\
\text { governance mobilization }\end{array}$ & $\begin{array}{c}\text { GOVERNMENT } \\
\text { INFORMATION QUARTERLY }\end{array}$ & 2012 \\
\hline Wan, PYZ. & $\begin{array}{l}\text { Analytical Sociology: A Bungean } \\
\text { Appreciation }\end{array}$ & SCIENCE \& EDUCATION & 2012 \\
\hline Joseph, RC. & $\begin{array}{c}\text { A structured analysis of e- } \\
\text { government studies: Trends and } \\
\text { opportunities }\end{array}$ & $\begin{array}{c}\text { GOVERNMENT } \\
\text { INFORMATION QUARTERLY }\end{array}$ & 2013 \\
\hline Kassen, M. & $\begin{array}{c}\text { A promising phenomenon of open } \\
\text { data: A case study of the Chicago open } \\
\text { data project }\end{array}$ & $\begin{array}{c}\text { GOVERNMENT } \\
\text { INFORMATION QUARTERLY }\end{array}$ & 2013 \\
\hline Não Identificado & $\begin{array}{c}\text { ELECTRONIC GOVERNMENT: a study } \\
\text { on the possibilities for citizen } \\
\text { participation }\end{array}$ & $\begin{array}{l}\text { INFORMACAO \& } \\
\text { SOCIEDADE-ESTUDOS }\end{array}$ & 2013 \\
\hline $\begin{array}{c}\text { Janssen, } \\
\text { M.; Estevez, E. }\end{array}$ & $\begin{array}{l}\text { Lean government and platform-based } \\
\text { governance-Doing more with less }\end{array}$ & $\begin{array}{c}\text { GOVERNMENT } \\
\text { INFORMATION QUARTERLY }\end{array}$ & 2013 \\
\hline Linders, D. & $\begin{array}{l}\text { From e-government to we- } \\
\text { government: Defining a typology for } \\
\text { citizen coproduction in the age of } \\
\text { social media }\end{array}$ & $\begin{array}{l}\text { Government Information } \\
\text { Quarterly }\end{array}$ & 2013 \\
\hline Bunge, $\mathrm{M}$. & $\begin{array}{c}\text { BIG QUESTIONS COME IN BUNDLES, } \\
\text { HENCE THEY SHOULD BE TACKLED } \\
\text { SYSTEMICALLY }\end{array}$ & $\begin{array}{l}\text { INTERNATIONAL JOURNAL } \\
\text { OF HEALTH SERVICES }\end{array}$ & 2014 \\
\hline $\begin{array}{c}\text { Ochara, } \\
\text { NM.; Mawela, T. }\end{array}$ & $\begin{array}{c}\text { Enabling Social Sustainability of E- } \\
\text { Participation through Mobile } \\
\text { Technology }\end{array}$ & $\begin{array}{l}\text { INFORMATION } \\
\text { TECHNOLOGY FOR } \\
\text { DEVELOPMENT }\end{array}$ & 2015 \\
\hline
\end{tabular}




\begin{tabular}{|c|c|c|c|}
\hline Li, MN.; Mao, JY. & $\begin{array}{l}\text { Hedonic or utilitarian? Exploring the } \\
\text { impact of communication style } \\
\text { alignment on user's perception of } \\
\text { virtual health advisory services }\end{array}$ & $\begin{array}{l}\text { INTERNATIONAL JOURNAL } \\
\text { OF INFORMATION } \\
\text { MANAGEMENT }\end{array}$ & 2015 \\
\hline $\begin{array}{c}\text { Grabner, } \\
\text { C.; Kapeller, J. }\end{array}$ & $\begin{array}{l}\text { New Perspectives on Institutionalist } \\
\text { Pattern Modeling: Systemism, } \\
\text { Complexity, and Agent-Based } \\
\text { Modeling }\end{array}$ & $\begin{array}{l}\text { JOURNAL OF ECONOMIC } \\
\text { ISSUES }\end{array}$ & 2015 \\
\hline $\begin{array}{c}\text { Neis, D.; Pereira, } \\
\text { MF. }\end{array}$ & $\begin{array}{c}\text { THE ORIGIS OF THE FIELD OF } \\
\text { STRATEGY: CONTRIBUTIONS BY IGOR } \\
\text { ANSOFF AND HENRY MINTZBERG }\end{array}$ & $\begin{array}{l}\text { REVISTA ELETRONICA DE } \\
\text { ESTRATEGIA E NEGOCIOS- } \\
\text { REEN }\end{array}$ & 2015 \\
\hline $\begin{array}{l}\text { Cumbie, B.A., } \\
\text { Kar, B. }\end{array}$ & $\begin{array}{c}\text { The role of social media in U.S. county } \\
\text { governments: The strategic value of } \\
\text { operational aimlessness }\end{array}$ & $\begin{array}{l}\text { International Journal of } \\
\text { Electronic Government }\end{array}$ & 2015 \\
\hline Barrance, $\mathrm{T}$. & $\begin{array}{c}\text { Framing Gov2.0: A Q-Methodological } \\
\text { Study of Practitioners' and Local } \\
\text { Politicians' Opinions }\end{array}$ & Local Government Studies & 2015 \\
\hline $\begin{array}{l}\text { Schulz, D., } \\
\text { Newig, J. }\end{array}$ & $\begin{array}{l}\text { Assessing online consultation in } \\
\text { participatory governance: Conceptual } \\
\text { framework and a case study of a } \\
\text { national sustainability-related } \\
\text { consultation platform in Germany }\end{array}$ & $\begin{array}{l}\text { Environmental Policy and } \\
\text { Governance }\end{array}$ & 2015 \\
\hline Teixeira, F. et al. & $\begin{array}{l}\text { Applying the semantic web to } \\
\text { represent an individual's academic and } \\
\text { professional background }\end{array}$ & $\begin{array}{l}\text { JOURNAL OF INFORMATION } \\
\text { SCIENCE }\end{array}$ & 2016 \\
\hline $\begin{array}{c}\text { de Brito, } \\
\text { AGC.; Quoniam, } \\
\text { L.; Mena-Chalco, } \\
\text { JP. }\end{array}$ & $\begin{array}{l}\text { Investigation of the Lattes Platform by } \\
\text { subject: A methodology proposal }\end{array}$ & TRANSINFORMACAO & 2016 \\
\hline $\begin{array}{l}\text { Bwalya, } \\
\text { KJ.; Mutula, S. }\end{array}$ & $\begin{array}{l}\text { A conceptual framework for e- } \\
\text { government development in resource- } \\
\text { constrained countries: The case of } \\
\text { Zambia }\end{array}$ & $\begin{array}{l}\text { INFORMATION } \\
\text { DEVELOPMENT }\end{array}$ & 2016 \\
\hline Hao, XL. et al. & $\begin{array}{c}\text { How to strengthen the social media } \\
\text { interactivity of e-government Evidence } \\
\text { from China }\end{array}$ & $\begin{array}{l}\text { ONLINE INFORMATION } \\
\text { REVIEW }\end{array}$ & 2016 \\
\hline $\begin{array}{l}\text { Ranerup, A. et } \\
\text { al. }\end{array}$ & $\begin{array}{c}\text { An analysis of business models in } \\
\text { Public Service Platforms }\end{array}$ & $\begin{array}{c}\text { GOVERNMENT } \\
\text { INFORMATION QUARTERLY }\end{array}$ & 2016 \\
\hline Klievink, B. et al. & $\begin{array}{c}\text { The collaborative realization of public } \\
\text { values and business goals: Governance } \\
\text { and infrastructure of public-private } \\
\text { information platforms }\end{array}$ & $\begin{array}{c}\text { GOVERNMENT } \\
\text { INFORMATION QUARTERLY }\end{array}$ & 2016 \\
\hline $\begin{array}{l}\text { Velasquez, } \\
\text { GG.; Oliveira, JP. }\end{array}$ & $\begin{array}{l}\text { General Theory of Systems and } \\
\text { Tourism: reflections and trajectory }\end{array}$ & $\begin{array}{l}\text { INVESTIGACIONES } \\
\text { TURISTICAS }\end{array}$ & 2016 \\
\hline Ma, BJ. et al. & $\begin{array}{c}\text { Semantic search for public opinions on } \\
\text { urban affairs: A probabilistic topic } \\
\text { modeling-based approach }\end{array}$ & $\begin{array}{l}\text { INFORMATION PROCESSING } \\
\text { \& MANAGEMENT }\end{array}$ & 2016 \\
\hline
\end{tabular}

Fonte: Os autores (2016)

Com base na análise e leitura do conteúdo das 25 referencias selecionadas, observou- 
se que $32 \%$ (8) dos artigos foram publicados durante o ano de 2016, na sequência, $28 \%$ (7) em 2015, 20\% (5) no ano de 2013, 8\% (2) em 2012 e 16\% (3) nos anos de 2010, 2011 e 2014 evidenciando um aumento de interesse no assunto que demonstra sua atualidade.

As bibliografias foram publicadas em periódicos científicos, ou seja, nenhuma delas publicadas em eventos científicos ou outros. Dentre os periódicos, o Government Information Quarterly obteve destaque com $28 \%$ (7) dos artigos publicados o que evidencia que essa fonte de informação é relevante para o assunto do ponto de vista da produção internacional.

Dos periódicos brasileiros, identificou-se a presença dos periódicos Transinformação e Informação \& Sociedade como os mais representativos sobre o assunto.

\section{CONSIDERAÇÕES FINAIS}

Por fim observou-se a relevância dos conteúdos disponíveis em cada artigo com relação às temáticas relacionadas de plataformas e-government, sistemismo e sistemas de informação e verificou-se que é possível extrair novas informações relacionadas ao tema para o desenvolvimento do assunto numa perspectiva interdisciplinar.

No entanto, pelo fato de ambas as bases de dados serem de amplitude internacional, constatou-se a falta de artigos nos quais a Plataforma Lattes é considerada o objeto de estudo e, não apenas um instrumento para a coleta de dados. Com isso, reforça-se a importância de estudos que investiguem o funcionamento e utilidade da plataforma com base na visão de agentes sociais que fazem parte da mesma, bem como agentes técnicos.

Por outro lado, os resultados alcançados permitem estabelecer marcos fundamentais sobre os periódicos, áreas de conhecimento e autores que fundamentam de algum ponto de vista a construção do conhecimento sobre o assunto numa perspectiva interdisciplinar e sistêmica, particularmente evidenciando critérios a serem levados em consideração em futuros estudos, quais sejam, as três Leis de Dados Abertos: i) se um dado não pode ser encontrado ou indexado na Web, então ele não existe; ii) se o dado não estiver aberto e disponível em formato compreensível por máquina, ele não pode ser reaproveitado; por fim, iii) se algum dispositivo legal não permitir sua replicação, então o dado não é útil (Eaves 2009).

Considerou-se que a pesquisa realizada permitiu identificar produção cientifica relativa ao assunto e reflexões relacionadas à temática abordada, concentrando-se em aspectos gerais e contribuindo para identificação de lacunas quanto à necessidade de se produzir conhecimento sobre os mecanismos que promovem e obstruem o funcionamento da Plataforma Lattes a partir de um ponto de vista do sujeito sistêmico, ou seja, que considera aspectos técnicos, mas também sociais a partir dos usuários, sendo essa uma oportunidade de pesquisa no campo da Ciência da Informação.

\section{REFERÊNCIAS}

ARAÚJO, C. A Correntes teóricas da ciência da informação. Ciência da Informação, Brasília, v. 38, n. 3, p. 192-204, set./dez. 2009. Disponível em: http://revista.ibict.br/ciinf/index.php/ciinf/article/view/1719/1347. Acesso em: 3 jun. 2016.

BERTALANFFY, L. Von. The theory of open systems in physics and biology. In: EMERY, F. E. Systems thinking. Londres: Penguin Books, 1969.

BORKO, H. Information Science: What is it? American Documentation, v. 19, n. 1, p. 3-5, jan. 1968. 
https://www.marilia.unesp.br/Home/Instituicao/Docentes/EdbertoFerneda/k---artigo-01.pdf. Acesso em: 10 mai. 2016.

BUNGE, M. A systemic perspective on crime. In: WIKSTRÖM, PerOlof H.; SAMPSON, R. J. The explanation of crime: context, 80 mechanisms, and development. Cambridge University Press, 2006, $\quad$ p. 8-30. Disponível em: https://www.researchgate.net/publication/288307258 A systemic perspective on crime. Acesso em: 18 mai. 2016.

BUNGE, M. Emergence and convergence: qualitative novelty and the unity of knowledge. Toronto: University of Toronto, 2003.

BUNGE, M. Mechanism and explanation. Philosophy of the social sciences, v. 27, n. 4, p. 410465, $1997 . \quad$ Disponível em: http://pos.sagepub.com/content/27/4/410.short?rss=1\&ssource=mfc. Acesso em: 18 mai. 2016.

BUNGE, M. Systemism: the alternative to individualism and holism. Journal of SocioEconomics, v. 29, n. 2, p. 147-157, 2000. Disponível em: http://www.sciencedirect.com/science/article/pii/S1053535700000585. Acesso em: 19 mai. 2016.

CASTELLS, Manuel. A Sociedade em Rede. Tradução de Roneide Venâncio Majer. São Paulo: Paz e Terra, 2005.

CÔRTES, P. L. Administração de Sistemas de Informação. São Paulo: Editora Saraiva, 2008.

EAVES, D. The three laws of open government, 2009 Disponível em: http://eaves.ca/. Acesso em: 8 jul. 2018.

FERRER, F; SANTOS, P. E-government: o governo eletrônico no Brasil. São Paulo: Saraiva, 2004.

HAWKINS, D.T; LARSON, S.E.; CATON, B.Q. Information science abstracts: tracking the literature of information science. Part 2: A new taxonomy for information science. Journal of the American Society for Information Science and Technology, v.54. n.8, p.771-781, 2003. Disponível em: http://onlinelibrary.wiley.com/doi/10.1002/asi.10275/pdf. Acesso em: 22 mai. 2016.

JAPIASSU, Hilton. Interdisciplinaridade e patologia do saber. Rio de Janeiro: Imago, 1976.

JARDIM, J. M. Capacidade governativa, informação e governo eletrônico. DataGramaZero, Rio de Janeiro, v. 1, n. 5, p. 1-8. out. 2000.

$\mathrm{KOH}, \mathrm{C}$. E.; RYAN, S.; PRYBUTOK, V. R. Creating value through managing knowledge in an egovernment to constituency $(\mathrm{G} 2 \mathrm{C})$ environment. The Journal of Computer Information Systems, v. 45, n. 4, p.32-41, 2005. Disponível em: http://www.tandfonline.com/doi/abs/10.1080/08874417.2005.11645853. Acesso em: 15 jul. 2016. 
MATSUDA, C. T. Governo eletrônico, a reforma democrática do estado-nação: a Prefeitura da cidade de São Paulo. 2006. Tese (Doutorado em Ciências da Comunicação) -Universidade de São Paulo, São Paulo, 2006.2 Disponível em http://www.teses.usp.br/teses/disponiveis/27/27148/tde-04082009-214906/pt-br.php.

Acesso em: 25 Jul. 2016.

MINAYO, M. C. de S. O desafio da pesquisa social. In: DESLANDES, Suely Ferreira; GOMES, Romeu; MINAYO, Maria Cecília de Souza (Org.). Pesquisa social: teoria, método e criatividade. 29. ed. Petrópolis: Vozes, 2010.

O'BRIEN, J. Sistemas de informação e as decisões gerenciais na era da internet. 2ed. São Paulo: Saraiva, 2004.

OLIVEIRA, J.F. Sistemas de informação versus tecnologia de informação: um impasse empresarial. São Paulo: Érica, 2004.

PACHECO, R. C. dos S.; KERN, V. M.; STEIL, A. V. Aplicações de arquitetura conceitual em plataformas e-gov: da gestão da informação pública à construção da sociedade do conhecimento. Ponto de Acesso, Salvador, v.1, n.1, p. 71-87, jun. 2007. Disponível em: http://www.portalseer.ufba.br/index.php/revistaici/article/viewFile/1394/883. Acesso em: 17 jul. 2016.

PACIFIC COUNCIL ON INTERNATIONAL POLICY. Roadmap for e-government in the developing world. Los Angeles: Pacific Council on International Policy, 2002. Disponível em: http://www.itu.int/net/wsis/docs/background/themes/egov/pacific council.pdf. Acesso em: 6 jun. 2016.

PINHEIRO, L.V.R. Campo interdisciplinar em ciência da informação: fronteiras remotas e recentes. Investigación Bibliotecológica, v.12, n.25, p.132-163, 1998. Disponível em: < http://www.revistas.unam.mx/index.php/ibi/article/view/3884>. Acesso em 25 mai. 2016.

REZENDE, D.A.; ABREU, A.F. Tecnologia da informação aplicada a sistemas de informação empresariais: o papel estratégico da informação e dos sistemas de informação nas empresas. 3. ed. São Paulo: Atlas, 2003.

SABBATINI, M. Lattes, o como gestionar la ciencia brasileña em la red. e-ciencia (Portal de Ciencia y Tecnología), 18 jun. 2001. Disponível em: http://www.galeon.com/divulcat/articu/141a.htm. Acesso em 22 mai. 2016.

SAMPAIO, R. F; MANCINI, M. C. Estudos de revisão sistemática: um guia para síntese criteriosa da evidência científica. Rev. bras. fisioter., 2007, vol.11, n.1, pp.83-89. Disponível em: http://www.scielo.br/pdf/rbfis/v11n1/12.pdf. Acesso em: 2 ago. 2016.

SILVA, P. M. O comportamento dos usuários de bibliotecas em sistemas de informação. TransInformação, Campinas, 20(3): 255-263, set./dez., 2008. Disponível em: http://periodicos.puc-campinas.edu.br/seer/index.php/transinfo/article/view/524. Acesso em: 4 junh. 2016.

STAIR, R.M.; REYNOLDS, G.W. Princípios de sistemas de informação: uma abordagem gerencial. 4.ed. Tradução Alexandre Melo de Oliveira. Rio de Janeiro: LTC, 2002.

Perspectivas em Gestão \& Conhecimento, João Pessoa, v. 9, n. 1, p. 198-211, jan./abr. 2019 
UNITED NATIONS. UN E-government survey 2016. E-government in Support of Sustainable Development. New York: Department of Economic and Social Affairs. Disponível em: https://doi.org/10.1016/S1369-7021(02)00629-6. Acesso em: 20 set. 2018.

VOLPATO, G. L. Ciência: da filosofia à publicação. 4. ed. rev. ampl. Botucatu, SP: Tipomic, 2004.

ZINS, C. Conceptions of Information Science. Journal of the American Society for Information Science and Technology, v. 58, n. 3, p. 335-350, 2007. Disponível em: < http://www.infolib.sk/files/Novy portal infolib subory/marta duskova/zins-conceptioninformation-science-pdf.pdf >. Acesso em: 3 jun. 2016. 